[Article]

\title{
功能化石墨烯/聚苯胺复合电极材料的制备和电化学性能
}

\author{
汪丽丽 邢瑞光 张邦文 ${ }^{*}$ 侯 渊 \\ ( 内蒙古科技大学稀土学院, 内蒙古包头 014010)
}

\begin{abstract}
摘要: 利用水合肼还原十八胺(ODA)接枝的氧化石墨烯(GO), 得到了十八胺功能化石墨烯(ODA-G), 将 ODA$G$ 与聚苯胺(PANI)通过溶液共混法, 制备了功能化石墨烯和聚苯胺纳米复合材料(ODA-G/PANI). 采用傅里叶 变换红外(FTIR)光谱、X射线衍射 (XRD)、热重分析(TGA)、拉曼(Raman)光谱及透射电镜(TEM), 对复合材料的 结构和形貌进行了表征; 利用循环伏安、恒流充放电及交流阻抗谱等, 对复合材料的电化学性能进行了测试. 结 果显示, 少量 ODA-G 的引入为PANI 的电化学氧化还原反应提供了更多的电子通道和活性位置, 有利于提高 PANI 的赝电容. 在电流密度 $1.0 \mathrm{~A} \cdot \mathrm{g}^{-1}$ 下, $2 \%(w)$ ODA-G/PANI 的比电容达到 $787 \mathrm{~F} \cdot \mathrm{g}^{-1}$, 而相应的 PANI 仅有 $426 \mathrm{~F} \cdot \mathrm{g}^{-1}$. 此外, ODA-G/PANI 的循环稳定性也远高于纯PANI.
\end{abstract}

关键词: 功能化石墨烯; 聚苯胺; 超级电容器; 电化学性能; 溶液混合

中图分类号: 0646

\section{Preparation and Electrochemical Properties of Functionalized Graphene/ Polyaniline Composite Electrode Materials}

\author{
WANG Li-Li XING Rui-Guang ZHANG Bang-Wen* HOU Yuan \\ (School of Rare Earth, Inner Mongolia University of Science \& Technology, Baotou 014010, \\ Inner Mongolia Autonomous Region, P. R. China)
}

\begin{abstract}
Octadecylamine functionalized graphene (ODA-G) was synthesized by the grafting of graphene oxide $(G O)$ with ODA followed by reduction with hydrazine hydrate. Subsequently, ODA-G/polyaniline (PANI) composites were prepared using a facile solvent-blending procedure. ODA-G and ODA-G/PANI composites were characterized by Fourier transform infrared spectrometry (FTIR), X-ray diffraction (XRD), thermogravimetric analysis (TGA), Raman spectroscopy, and transmission electron microscopy (TEM). The electrochemical properties of the composites were measured based on cyclic voltammetry (CV), galvanostatic charge/discharge, and ac impedance spectroscopy. The results show that ODA-G as a support material provides additional electron transfer paths, as well as active sites, for the electrochemical redox reaction of PANI, which helps to increase its pseudocapacitance. A specific capacitance of $782 \mathrm{~F} \cdot \mathrm{g}^{-1}$ is obtained for $2 \%(w)$ ODA-G/PANI at a current density of $1.0 \mathrm{~A} \cdot \mathrm{g}^{-1}$, compared with $426 \mathrm{~F} \cdot \mathrm{g}^{-1}$ for PANI. Furthermore, ODA-G/PANI exhibits better stability than PANI.
\end{abstract}

Key Words: Functionalized graphene; Polyaniline; Supercapacitor; Electrochemical property; Solvent-blending

Received: May 19, 2014; Revised: June 16, 2014; Published on Web: June 16, 2014.

"Corresponding author. Email: bangwenz@126.com. Tel: +86-472-6896876.

The project was supported by the National Natural Science Foundation of China (51164026), Inner Mongolia University of Science and Technology Innovation Fund, China (2012NCL007).

国家自然科学基金(51164026)和内蒙古科技大学创新基金(2012NCL007)资助项目

(C) Editorial office of Acta Physico-Chimica Sinica 


\section{1 引 言}

超级电容器作为一种介于电解质电容器与电 池之间的新型储能设备, 因其具有充电速度快、功 率密度高、循环寿命长等优点, 一直备受研究者关 注. 高性能超级电容器的获得主要依赖于高电容的 电极材料. ${ }^{1}$ 目前研究的电极材料主要有三类: 碳材 料、金属氧化物和导电聚合物. ${ }^{2}$ 碳材料具有导电性 好、力学性能佳、循环寿命长等特点, 但其电容较 小; 金属氧化物噟电容高, 但导电性差、成本高; 导 电聚合物的噟电容也较高、可加工性好, 但循环寿 命较低. 因此, 将碳材料 ${ }^{3-7}$ (如活性炭、碳纳米管、石 墨烯)与导电聚合物(如聚苯胺(PANI)、聚吡咯、聚噻 吩)复合, 制备综合性能优异的电极材料成为当前超 级电容器领域的研究热点之一 ${ }^{8}$ 其中, 对石墨烯/聚 苯胺体系的研究尤为注目.

目前,制备石墨烯/聚苯胺复合材料主要有两类 方法: 原位化学聚合法和电化学聚合法. Ram 等 ${ }^{9}$ 在 石墨烯溶液中引发苯胺单体聚合, 制备了石墨烯/聚 苯胺复合材料, 在 $0.1 \mathrm{~A} \cdot \mathrm{g}^{-1}$ 电流密度下, 测得比电容 为 $300-500 \mathrm{~F} \cdot \mathrm{g}^{-1}$. Yan 等 ${ }^{10}$ 采用类似的方法在石墨 烯纳米片表面生长出 $2 \mathrm{~nm}$ 大小的聚苯胺纳米颗粒, 在 $1 \mathrm{mV} \cdot \mathrm{s}^{-1}$ 的扫描速率下该材料比电容高达 $1046 \mathrm{~F}$. $\mathrm{g}^{-1}$. $\mathrm{Wu}$ 等 ${ }^{11}$ 首先原位聚合氧化石墨烯/聚苯胺前驱 体, 然后通过水合肼还原制备石墨烯/聚苯胺复合材 料, 测得比电容达 $480 \mathrm{~F} \cdot \mathrm{g}^{-1}$, 并且循环性能较好. 由 于石墨烯容易团聚, 为原位化学聚合法制备均匀的 石墨烯/聚苯胺复合材料带来一定难度, 为此一些研 究开始对石墨烯进行选择性功能化, 以便于石墨烯 的分散和原位聚合. Liu等 ${ }^{12}$ 采用草酸对氧化石墨烯 功能化, 得到羧基化氧化石墨烯, 然后再与苯胺原 位化学聚合, 制得的电极复合材料比电容可达 525 $\mathrm{F} \cdot \mathrm{g}^{-1}$. Shen 等 ${ }^{13}$ 用氨基硅烷接枝石墨烯, 然后原位聚 合聚苯胺, 所得电极复合材料比电容高达 $500 \mathrm{~F}$. $\mathrm{g}^{-1}$. 在电化学聚合方面, $\mathrm{Li}$ 等 ${ }^{14}$ 以石墨烯片为阳极电 聚合聚苯胺, 得到的复合材料抗拉强度达到 12.6 $\mathrm{MPa}$, 比电容为 $233 \mathrm{~F} \cdot \mathrm{g}^{-1}$. Zhang 等 ${ }^{15}$ 采用电沉积法 制备了氧化石墨烯/聚苯胺复合材料, 获得了较高的 比电容, 1000 次循环保持率达到 89\%. 此外,一些其 它复合路线也有报道. ${ }^{16}$

上述研究尽管取得了不同程度的成功, 但其反 应条件不易控制, 复合材料配方设计不便, 不适合 电极材料的大规模制备, 寻求简便高效的制备路线 是学界的热切期望. 为此, 本文报道了制备石墨烯
与聚苯胺电极复合材料的一条新路线, 通过十八胺 (ODA)功能化解决石墨烯的分散性问题, 通过简单 的溶液混合取代复杂的溶液聚合, 获得高性能的超 级电容器电极材料.

\section{2 实验部分}

\section{1 试剂与仪器}

天然鳞片石墨(399 目, 青岛美利坤石墨制备 厂), 苯胺、高锰酸钾、过硫酸钾、过硫酸铵、氯仿、 $N$ 甲基吡咯烷酮(AR, 天津市风船化学试剂有限公 司), Super P Li、KS6(电子级, 太原市迎泽区力之源 电池销售部), 无水乙醇(AR, 天津市永大化学试剂 有限公司), 浓硫酸(AR, 北京化工厂), 五氧化二磷 (AR, 北京化学试剂公司), $30 \%(w)$ 双氧水(AR, 天津 市华东试剂厂).

本实验使用 $\mathrm{X}$ 射线衍射仪 $\left(X R D, X^{\prime}\right.$ Pert PRO, PANalytical) 鉴别粉体物相, 铜靶 $\mathrm{Cu} K_{a}$ 辐射 $(\lambda=$ $0.154 \mathrm{~nm})$; 粉体样品经研磨后与 $\mathrm{KBr}$ 混合压片, 采 用傅里叶变换红外光谱仪(FTIR-N3896, PerkinElmer) 和拉曼光谱仪(LabRAM XploRA, HORIBA JobinYvon S.A.S)鉴定其分子结构; 采用综合热分析仪 (SDT Q600, TA) 分析样品失重, 氩气保护, 升温速度 $10{ }^{\circ} \mathrm{C} \cdot \mathrm{min}^{-1}$; 以铜网为载具, 采用透射电子显微镜 (TEM, JEM-2000FX, JEOL) 观察粉体形貌, 加速电 压 $200 \mathrm{kV}$. 采用电化学工作站(PARSTAT2273, AMETEK)测试电极材料的电化学性能, 测试系统为三 电极体系, 工作电极为待测电极, 辅助电极为铂片, 参比电极为饱和甘录电极 $(\mathrm{SCE})$, 电解液为 $1 \mathrm{~mol} \cdot \mathrm{L}^{-1}$ 的 $\mathrm{H}_{2} \mathrm{SO}_{4}$ 溶液.

\subsection{ODA-G/PANI复合材料的制备}

\subsubsection{ODA-G 的制备}

采用改进的 Hummers 法制备氧化石墨烯(GO). 其简要过程如下, 将浓硫酸加热到 $80^{\circ} \mathrm{C}$, 加入过硫 酸钾和五氧化二磷, 然后加入天然鳞片石墨反应, 达到预氧化目的; 之后, 在浓硫酸冰浴中加入预氧 化石墨, 再加入高锰酸钾, 在 $35^{\circ} \mathrm{C}$ 反应 $4 \mathrm{~h}$, 然后加 入去离子水, 保证温度不超过 $50{ }^{\circ} \mathrm{C}$; 最后, 加入 $30 \%(w)$ 的过氧化氢溶液, 产生亮黄色的溶液, 过滤, 洗涤, 备用. 称取 $0.26 \mathrm{~g} \mathrm{GO}$ 溶于 $100 \mathrm{~mL}$ 的去离子 水, 超声 $1 \mathrm{~h}$; 称取 $0.42 \mathrm{~g}$ ODA 溶于 $30 \mathrm{~mL}$ 的无水乙 醇. ${ }^{17}$ 将以上两种溶液转移到三口烧瓶中, 在机械摚 拌下 $90^{\circ} \mathrm{C}$ 水浴回流 $10 \mathrm{~h}$, 加入适量水合肼继续回流 $10 \mathrm{~h}$. 之后, 用平均孔径为 $0.22 \mu \mathrm{m}$ 的聚丙烯过滤膜 
抽滤, 用无水乙醇洗涤数次, 干燥 $24 \mathrm{~h}$, 得到 $0.356 \mathrm{~g}$ 的十八胺功能化石墨烯(ODA-G).

\subsection{2 未功能化石墨烯 $(\mathrm{G})$ 的制备}

称取 $0.26 \mathrm{~g} \mathrm{GO}$ 溶于 $100 \mathrm{~mL}$ 的去离子水中, 超 声 $1 \mathrm{~h}$; 将溶液转移到三口烧瓶中, 在机械搅拌下 $90{ }^{\circ} \mathrm{C}$ 水浴回流 $10 \mathrm{~h}$, 加入适量水合肼继续回流 10 h. $20 \mathrm{~h}$ 后, 用平均孔径为 $0.22 \mu \mathrm{m}$ 的聚丙烯过滤膜抽 滤, 用无水乙醇洗涤数次, 干燥 $24 \mathrm{~h}$, 得到 $\mathrm{G}$.

\subsubsection{PANI的制备}

将三口烧瓶中加入 $50 \mathrm{~mL} 1.5 \mathrm{~mol} \cdot \mathrm{L}^{-1}$ 盐酸和 $2.3 \mathrm{~mL}$ 苯胺溶液, 冰浴下搅拌, 待温度降至 $5{ }^{\circ} \mathrm{C}$ 以 下, 缓慢滴加 $12.5 \mathrm{~mL} 2 \mathrm{~mol} \cdot \mathrm{L}^{-1}$ 过硫酸铵水溶液(过 硫酸铵与苯胺的摩尔比为 $1: 1)$. 反应 5-6 h 后, 将反 应产物减压抽滤, 分别用去离子水和无水乙醇洗涤 至滤液无色, 在 $60^{\circ} \mathrm{C}$ 真空干燥 $12 \mathrm{~h}$, 得到呈墨绿色 的粉末状 PANI.

\subsubsection{ODA-G/PANI 或 G/PANI 复合材料的制备}

将一定质量的 ODA-G(或 $\mathrm{G}$ )溶于氯仿中, 按一 定质量比称取 PANI 于 $N$-甲基吡咯烷酮中, 各超声 $30 \mathrm{~min}$. 将两种液体混合, 超声 $1 \mathrm{~h}$ 得到均匀的 ODAG/PANI(或 G/PANI)分散液.

\subsection{ODA-G/PANI 或 G/PANI 复合材料电极的}

\section{制备}

将活性物质(ODA-G/PANI 或 G/PANI)、导电剂 (Super P Li 与 KS6 质量比为 1.5 的混合导电剂)、粘 结剂(聚四氟乙烯(PTFE)乳液)按质量比 75:20:5 分散 于无水乙醇中, 在 $65^{\circ} \mathrm{C}$ 超声混合成塑性浆料, 然后 涂覆于不锈钢集流体并以 $15 \mathrm{MPa}$ 压力压片, 最后 $80{ }^{\circ} \mathrm{C}$ 真空干燥 $12 \mathrm{~h}$, 得待测电极.

\section{3 结果与讨论}

\section{1 材料的表征}

\subsubsection{FTIR 分析}

图 1 为 $\mathrm{GO}$ 与 $\mathrm{ODA}-\mathrm{G}$ 的红外光谱图. GO 在 $3000-3500 \mathrm{~cm}^{-1}$ 处出现一比较宽的吸收峰, 这是由 氧化石墨烯表面的 $\mathrm{OH}$ 引起的, 在 1733、1626 和 $1220 \mathrm{~cm}^{-1}$ 处的吸收峰分别代表了羧基上 $\mathrm{C}=\mathrm{O}$ 的伸 缩振动、芳环上的 $\mathrm{C}=\mathrm{C}$ 和 $\mathrm{C}-\mathrm{OH}$ 伸缩, $1050 \mathrm{~cm}^{-1}$ 处的吸收峰代表了环氧键上的 $\mathrm{C}-\mathrm{O}-\mathrm{C}$ 特征吸 收. ${ }^{18}$ 与 $\mathrm{GO}$ 的红外光谱对比, $\mathrm{ODA}-\mathrm{G}$ 在 $1733 \mathrm{~cm}^{-1}$ 处 的羧基吸收峰减弱甚至消失, 在 1030-1050 $\mathrm{cm}^{-1}$ 处 的环氧键吸收峰也明显减弱, 说明氧化石墨烯已经 被还原转变成为石墨烯; 而在 $1662 \mathrm{~cm}^{-1}$ 附近出现一

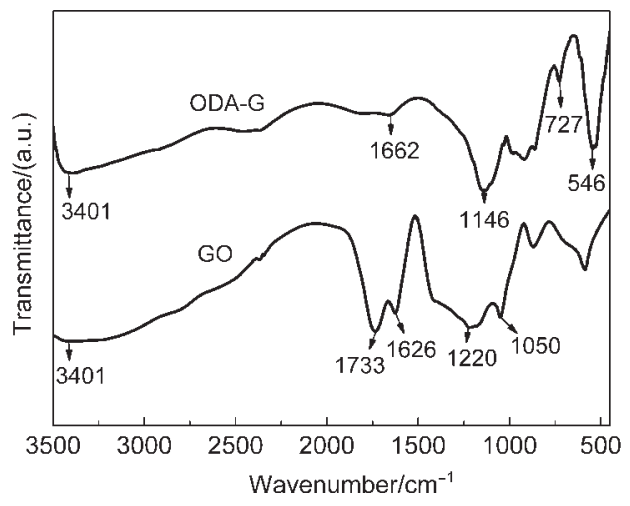

图 1 GO 和 ODA-G 的 FTIR 光谱图

Fig.1 FTIR spectra of GO and ODA-G

GO: graphene oxide; ODA-G: octadecylamine (ODA) functionalized graphene $(\mathrm{G})$

个新的吸收峰, 此峰为 $\mathrm{C}-\mathrm{NH}-\mathrm{C}$ 特征吸收峰, 这 说明还原的石墨烯已被十八胺功能化.

\subsubsection{TGA 分析}

图 2 为 $\mathrm{GO}$ 和 $\mathrm{ODA}-\mathrm{G}$ 的热重曲线. $\mathrm{GO}$ 在室 温 $-800{ }^{\circ} \mathrm{C}$ 范围内表现为三步失重, 第一步失重在 室温 $-150^{\circ} \mathrm{C}$, 失重 $11.11 \%$, 主要是吸附水的损失; 第二步失重在 $220-340{ }^{\circ} \mathrm{C}$, 失重 $32.45 \%$, 主要是氧 化石墨烯中氧以二氧化碳、一氧化碳和水的形式挥 发掉. 第三步失重在 340-800 ${ }^{\circ} \mathrm{C}$, 主要是石墨烯碳 的反应; ${ }^{19} \mathrm{ODA}-\mathrm{G}$ 在室温 $-800{ }^{\circ} \mathrm{C}$ 范围内两步失重, 第一步在 $210-340{ }^{\circ} \mathrm{C}$, 失重 $15 \%$, 认为是由石墨烯 中未发生反应的含氧基团的损失造成的, 第二步失 重在 $340-545{ }^{\circ} \mathrm{C}$, 失重 $45.48 \%$, 认为是接枝的十八 烷基链的损失, 故 ODA 的质量接枝率为 $45.48 \%$.

\subsubsection{XRD 分析}

图 3 为 GO、ODA-G、PANI 及 ODA-G/PANI 的 $\mathrm{XRD}$ 图. GO 在 $2 \theta \approx 11^{\circ}$ 处出现了与文献 ${ }^{18}$ 报道相符 的 GO 的特征衍射峰, 对应的晶面间距约为 0.78

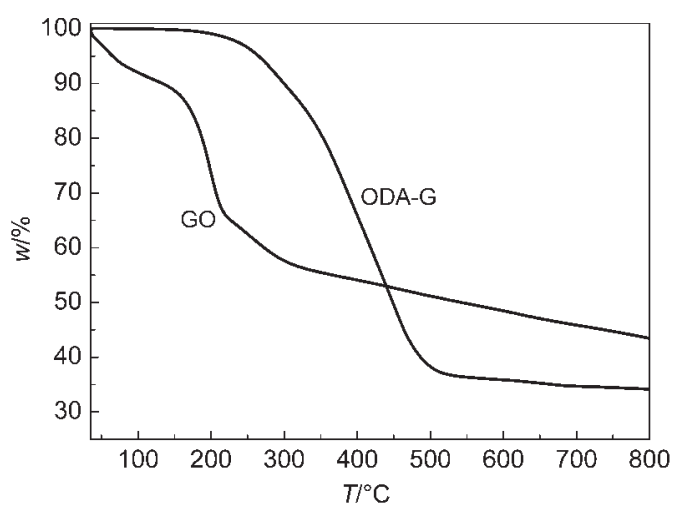

图 2 GO 和 ODA-G 的热重分析图

Fig.2 TG analyses of GO and ODA-G 


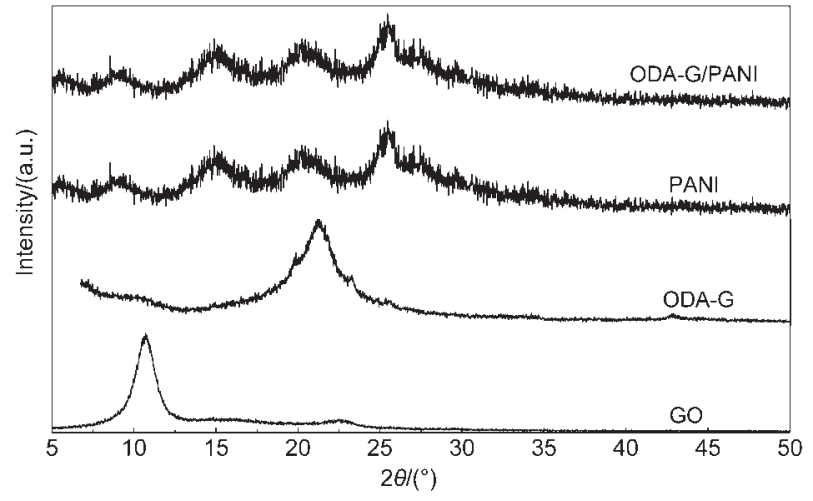

图 3 GO、ODA-G、PANI 及 ODA-G/PANI 的 XRD 图

Fig.3 XRD patterns of GO, ODA-G, PANI, and ODA-G/PANI

PANI: polyaniline

$\mathrm{nm}$, 说明本文制得了在石墨片层上含有大量氧化官 能团的 GO. GO 经 ODA 改性并被水合肼还原后, 其 特征衍射峰消失, 在 $21.3^{\circ}$ 出现一接近于石墨的特征 衍射峰, 但强度相对较弱, 峰形较宽, 这主要是由于 水合肼对 $\mathrm{GO}$ 的还原使得氧化官能团大量减少, 还 原后得到的石墨烯片趋向于恢复石墨的片层结构, 故在 $21.3^{\circ}$ 出现一接近于石墨的特征衍射峰 ${ }^{20}$ 而经 ODA 改性的石墨烯片层上接枝有大量的十八烷基, 十八烷基的引入使得石墨烯片不能发生团聚而恢 复到石墨的结构, 故峰强度相对较弱, 峰形较宽.

盐酸掺杂 PANI 在 $2 \theta$ 为 $9.88^{\circ} 、 14.46^{\circ} 、 20.18^{\circ}$ 、 $25.5^{\circ}$ 附近出现了与文献 ${ }^{21}$ 报道吻合的 PANI 的特征 衍射峰, 说明 PANI有一定的结晶度, 但是由于分子 链上刚性苯环的存在导致有序的结晶结构并不完 善, 故峰形较宽、强度较弱, 由此可知, 此 PANI 也呈 一定的无定形态. 而从 ODA-G/PANI 复合材料的 $\mathrm{XRD}$ 谱可以看出, 其与 PANI 的 XRD 谱基本相同, 这主要是由于 ODA-G 加入量相对较少, 被主相 PANI的衍射峰掩盖.

\subsubsection{Raman 光谱分析}

图 4 为 GO、ODA-G、ODA-G/PANI 的 Raman 光 谱. 在碳材料的 Raman 光谱中, $D$ 峰代表 $s p^{3}$ 杂化碳 原子的特征峰或由石墨烯边缘缺陷与无序化诱导 产生的 $s p^{2}$ 杂化缺陷的特征峰, $G$ 峰代表碳原子有序 $s p^{2}$ 杂化缺陷的特征峰. ${ }^{2}$ 天然石墨由于其具有规整 的结构, 故石墨晶格的面内振动引起的 $G$ 峰位于 $1580 \mathrm{~cm}^{-1}$ 处且窄而强. ${ }^{23} \mathrm{GO}$ 的 Raman 光谱显示, 当 大量含氧官能团的引入而产生缺陷形成 $\mathrm{GO}$ 时, 在 $1344 \mathrm{~cm}^{-1}$ 处出现 $D$ 峰, 说明 $s p^{2}$ 区域尺寸减小, 缺陷 区域增多; 而 $G$ 峰比天然石墨更宽, 偏离 $1580 \mathrm{~cm}^{-1}$,

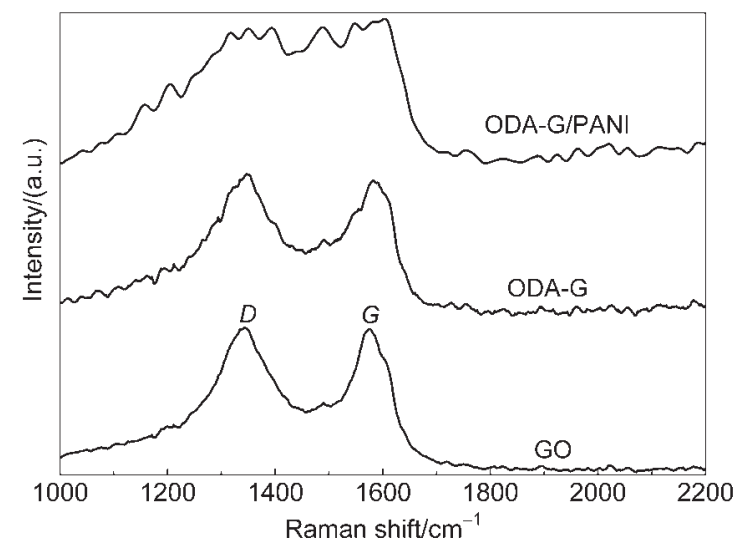

图 4 GO、ODA-G、ODA-G/PANI 的拉曼光谱 Fig.4 Raman spectra of GO, ODA-G, and ODA-G/PANI

说明有序 $s p^{2}$ 杂化碳结构转化成为 $s p^{3}$ 杂化碳结构, 即 $\mathrm{C}=\mathrm{C}$ 双键被破坏, $D$ 峰和 $G$ 峰强度之比 $\left(I_{D} / I_{G}\right)$ 为 3.25 , 同样也说明本文 GO 的成功制备. ODA-G 的 Raman 光谱显示, $G$ 峰接近于天然石墨的 $1580 \mathrm{~cm}^{-1}$, 说明 $s p^{2}$ 杂化碳结构被部分修复, 这与 XRD 中 ODA$\mathrm{G}$ 的特征衍射峰接近于石墨的特征衍射峰相吻合; $I_{D} / I_{G}=2.89$ 较 $\mathrm{GO}$ 的 $I_{D} / I_{G}=3.25$ 有所降低, 说明部分 $s p^{3}$ 杂化碳结构转变为 $s p^{2}$ 杂化碳结构. 同时由于有十八 胺的功能化导致较难恢复到三维的石墨结构, 所以 峰型较宽. ODA-G/PANI 的 Raman 光谱显示, 聚苯胺 在 $1163 \mathrm{~cm}^{-1}$ 处的 $\mathrm{C}-\mathrm{H}$ 面内弯曲振动, $1324 \mathrm{~cm}^{-1}$ 处 的 $\mathrm{C}-\mathrm{N}$ 伸缩振动, $1464 \mathrm{~cm}^{-1}$ 处的醌式 $\mathrm{C}=\mathrm{N}$ 伸缩振 动, $1598 \mathrm{~cm}^{-1}$ 处的苯式 $\mathrm{C}-\mathrm{C}$ 伸缩振动产生的特征 峰在 ODA-G/PANI 中部分发生了偏移, 如, $1464 \mathrm{~cm}^{-1}$ 蓝移至 $1488 \mathrm{~cm}^{-1}, 1598 \mathrm{~cm}^{-1}$ 蓝移至 $1604 \mathrm{~cm}^{-1}$; 而 ODA-G 的 $D$ 峰和 $G$ 峰在 ODA-G/PANI 不太明显, 这 主要是由于 ODA-G 加入量相对较少不能形成有效 的 $D$ 峰和 $G$ 峰. ${ }^{24,25}$

\subsubsection{TEM 分析}

图 5 为 PANI, ODA-G 及 ODA-G/PANI 的 TEM 图. 图 5(a)表明, 合成的 PANI 呈现出纤维状, 纤维直 径为 $200 \mathrm{~nm}$ 左右, 经插图放大后发现 PANI 表面长 满了小刺, 这种特殊的结构有利于增大 PANI 的比 表面积和噟电容. 图 5(b)表明, ODA-G 为透明的薄 层, 由于表面褶皱和卷曲, 使一些地方呈现深色. 图 5(c)为 ODA-G/PANI 复合材料的局部结构, 其中的 箭头指示 ODA-G. 可以观察到, 虽然石墨烯薄片含 量较少, 但由于 ODA-G 具有良好的分散性, 因此与 纳米 PANI复合后, 石墨烯没有明显的团聚, 而是较 好地嵌入到纳米 PANI 之间或包裹在纳米 PANI 表 

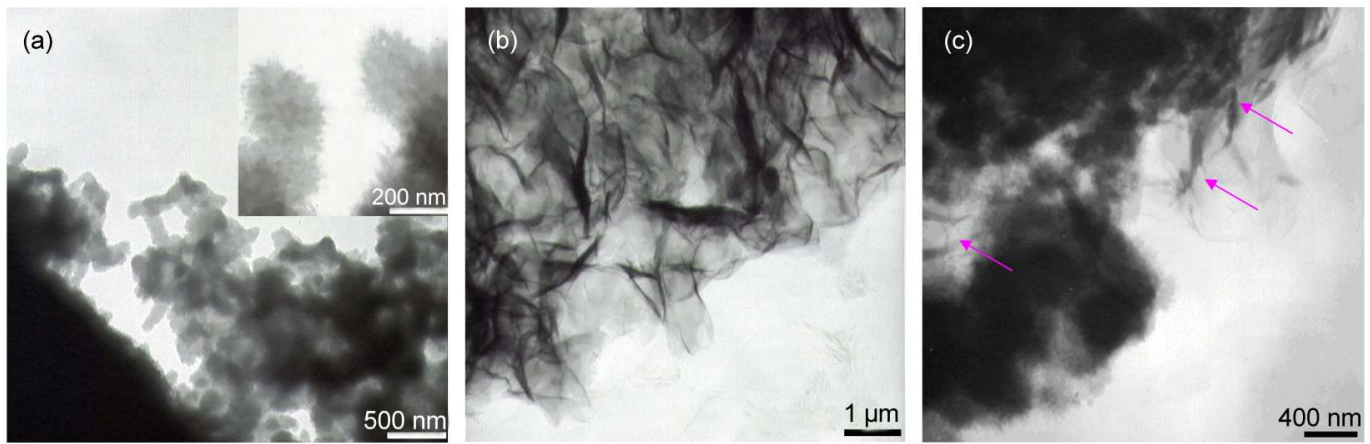

图 5 PANI (a)、ODA-G (b)及 ODA-G/PANI (c) 的 TEM 图

Fig.5 TEM images of PANI (a), ODA-G (b), and ODA-G/PANI (c)

面, 结合良好, 这对提高复合材料电化学性能的循 环稳定性十分重要.

\subsection{ODA-G/PANI复合材料的电化学性能}

图 6 为纯 PANI 及 $\mathrm{G} / \mathrm{PANI}$ 、不同 $\mathrm{ODA}-\mathrm{G}$ 含量的 ODA-G/PANI 复合材料在 $10 \mathrm{mV} \cdot \mathrm{s}^{-1}$ 的扫描速率下 的循环伏安曲线. 由图可知, 纯 PANI与不同 ODA-G 含量的 ODA-G/PANI 氧化还原峰的位置相同, 整体 形状大致相似, 说明电极材料的氧化还原峰主要来 源于聚苯胺的氧化还原反应, 是由聚苯胺的完全还 原态、部分氧化还原态和完全氧化态之间的相互转 化提供的. 而 G/PANI 的循环伏安曲线的形状相对 于纯 PANI、ODA-G/PANI 体系有所改变, 说明其的 加入会对 PANI 的氧化还原反应造成影响. 对于 ODA-G/PANI 复合材料来说, 随着 ODA-G 含量的增 加, 氧化还原峰对应的电位基本没有改变, 而从电 位位置可知, 这对氧化还原峰是由 PANI 的氧化还 原反应提供的, ODA-G 没有发生氧化还原反应, 即 ODA-G 几乎不提供噟电容.

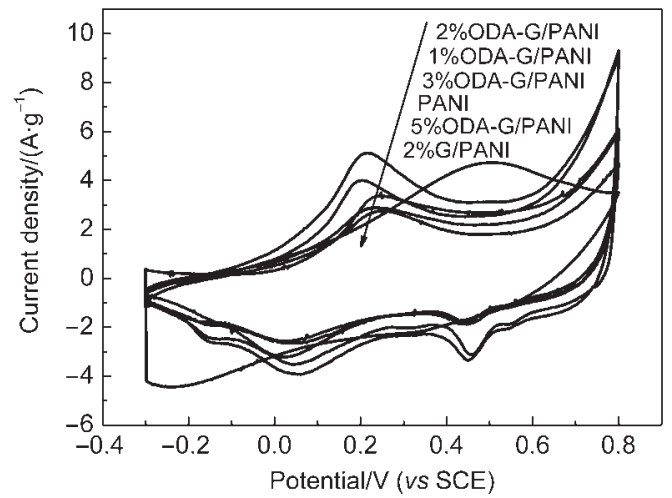

图 6 纯 PANI、 $2 \%(w)$ G/PANI 及不同含量 ODA-G 的ODAG/PANI 复合材料在 $10 \mathrm{mV} \cdot \mathrm{s}^{-1}$ 扫描速率时的循环伏安曲线

Fig.6 Cyclic voltammogram curves of PANI, $2 \%(w)$ G/ PANI, and ODA-G/PANI composites with different contents of ODA-G at a scanning rate of $10 \mathrm{mV} \cdot \mathrm{s}^{-1}$
图 7(A)为纯 PANI 及 G/PANI、ODA-G/PANI 复 合材料在 $1 \mathrm{~A} \cdot \mathrm{g}^{-1}$ 电流密度下的恒流充放电曲线, 图 7(B)为相应的比电容值的柱状图. 由恒流充放电曲 线计算比电容 $\left(C_{m}\right)$ 的公式 ${ }^{26}$ 如下所示:

$$
C_{m}=\frac{I \cdot \Delta t}{m \cdot \Delta V}
$$

式中, $m$ 为电极材料的质量 $(\mathrm{g}) ; I$ 为放电电流( $(\mathrm{A}) ; \Delta t$ 为放电时间 $(\mathrm{s}) ; \Delta V$ 是电压区间 $(\mathrm{V})$.

图 7(A)显示, 电极材料都具有良好的充放电特
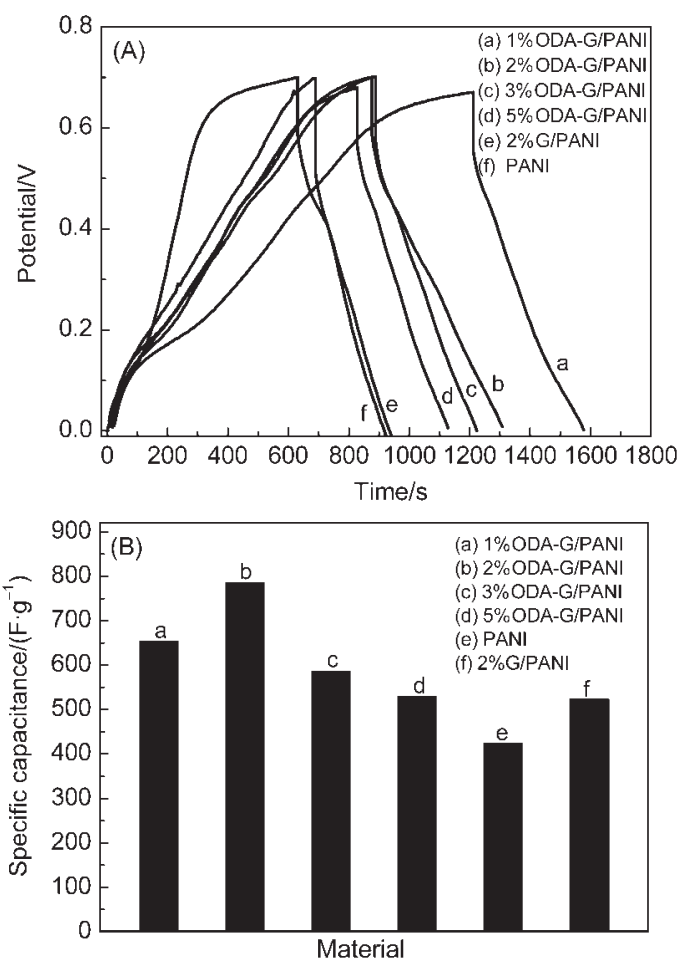

图 7 纯 PANI、 $2 \%(w) G / P A N I 、 O D A-G / P A N I$ 复合材料在电 流密度为 $1.0 \mathrm{~A} \cdot \mathrm{g}^{-1}$ 时的恒流充放电曲线(A)及其 比电容值柱状图(B)

Fig.7 Galvanostatic charge-discharge curves of PANI, 2\% (w)G/PANI, and ODA-G/PANI composites with different contents of ODA-G (A) and column graph of specific capacitance (B) obtained at a current density of $1.0 \mathrm{~A} \cdot \mathrm{g}^{-1}$ 
性. 依据上述公式可求图 7(A)的充放电曲线对应比 电容为 $1 \% \mathrm{ODA}-\mathrm{G} / \mathrm{PANI}: 654 \mathrm{~F} \cdot \mathrm{g}^{-1} ; 2 \% \mathrm{ODA}-\mathrm{G} /$ PANI: $787 \mathrm{~F} \cdot \mathrm{g}^{-1} ; 3 \%$ ODA-G/PANI: $588 \mathrm{~F} \cdot \mathrm{g}^{-1} ; 5 \%$ ODA-G/PANI: $530 \mathrm{~F} \cdot \mathrm{g}^{-1}$; PANI: $426 \mathrm{~F} \cdot \mathrm{g}^{-1} ; 2 \%(w) \mathrm{G} /$ PANI: $524 \mathrm{~F} \cdot \mathrm{g}^{-1}$ (图 7(B)). 由此可知, 纯 PANI 与 $\mathrm{G} /$ PANI 的比电容均小于 ODA-G/PANI 的比电容, 这主 要归因于 ODA-G 的引入为 PANI 提供了弥散而优 良的导电通道网络, 使得 PANI 发生氧化还原反应 时产生的电荷能迅速迁移并有效导出, 从而较大提 升了 PANI 的氧化还原反应效率, 使其具有更加优 异的比电容值. 相比而言, $\mathrm{G}$ 本身容易团聚难以形成 有效的导电通道网络, 故其引入并未显著提高比电 容值. 另外, ODA-G/PANI 的比电容随 ODA-G 加入 量的增加呈现出先增大后降低的趋势, 当 ODA-G 含量为 $2 \%$ 时电容达到最大, 这可能是因为: 当 ODA-G 含量小于 $2 \%$ 时, ODA-G 含量少, 未能形成 足够的电荷传输通道, 造成比电容值偏小; 当 ODA$\mathrm{G}$ 含量大于 $2 \%$ 时, 一方面, 过多的 ODA-G 在材料内 部局部团聚, 反而阻碍电荷传输, 另一方面, 对比电 容贡献有限的 ODA-G 会减少对噟电容贡献大的 PANI 所占含量.

图 8 为 $2 \%(w)$ ODA-G/PANI 复合材料在不同扫 描速率下的循环伏安曲线. 从图 8 可以看出, 随着扫 描速率的改变, 循环伏安曲线均出现两对氧化还原 峰, 并且峰的位置没有发生改变, 曲线的整体形状 基本不变, 呈现出大体的矩形特征, 这说明了复合 材料具有良好的稳定性; 当扫描速率增加到 $50 \mathrm{mV}$. $\mathrm{s}^{-1}$ 时, 曲线在 0.4-0.6 V 之间的氧化峰变得不明显, 主要由于扫描速率过大, 电极材料来不及发生反 应, 未产生相应的响应电流造成的.

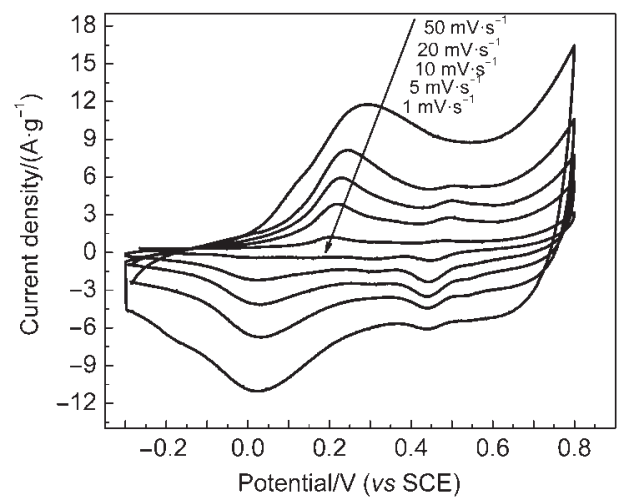

图 $82 \%(w)$ ODA-G/PANI 复合材料在不同扫描速率 下的循环伏安曲线

Fig.8 Cyclic voltammogram curves of $2 \%(w) O D A-G /$ polyaniline composite at different scan rates
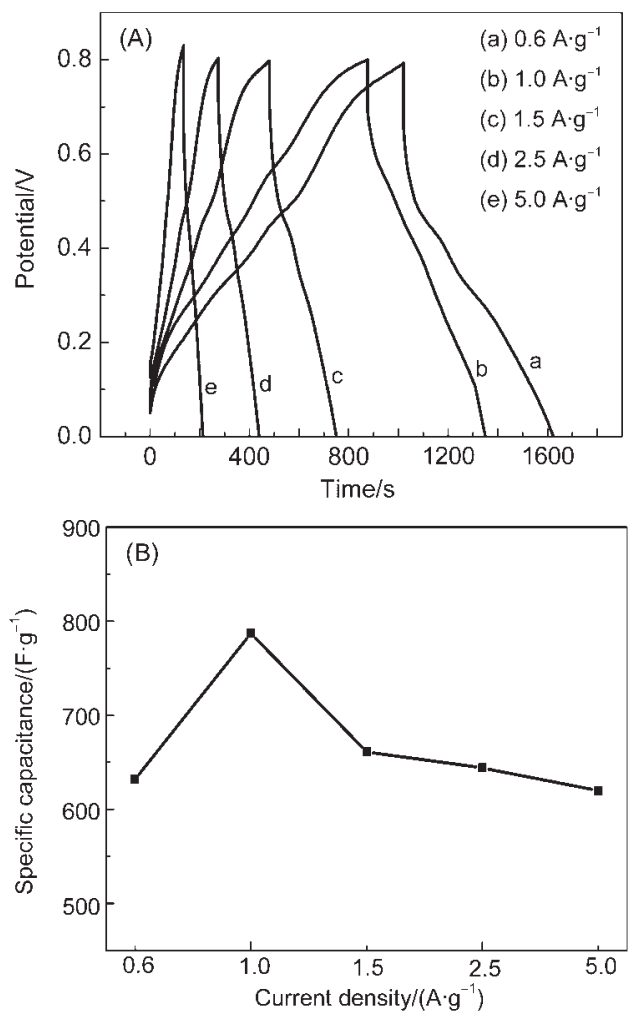

图 $92 \%(w)$ ODA-G/PANI 复合材料在不同电流密度时的恒 流充放电曲线(A)及其比电容值曲线图(B)

Fig.9 Galvanostatic charge-discharge curves (A) and curve graph of specific capacitance (B) of $2 \%(w)$ ODA-

G/PANI composite under different current densities

图 9 为 $2 \%(w)$ ODA-G/PANI 复合材料在不同电 流密度时的恒流充放电曲线及其比电容值曲线图. 从图 9 可知, $2 \%(w) \mathrm{ODA}-\mathrm{G} / \mathrm{PANI}$ 复合材料在不同的 电流密度下都表现出良好的充放电特性, 随电流密 度的增加比电容值降低, 但电流密度增加到 $5 \mathrm{~A} \cdot \mathrm{g}^{-1}$

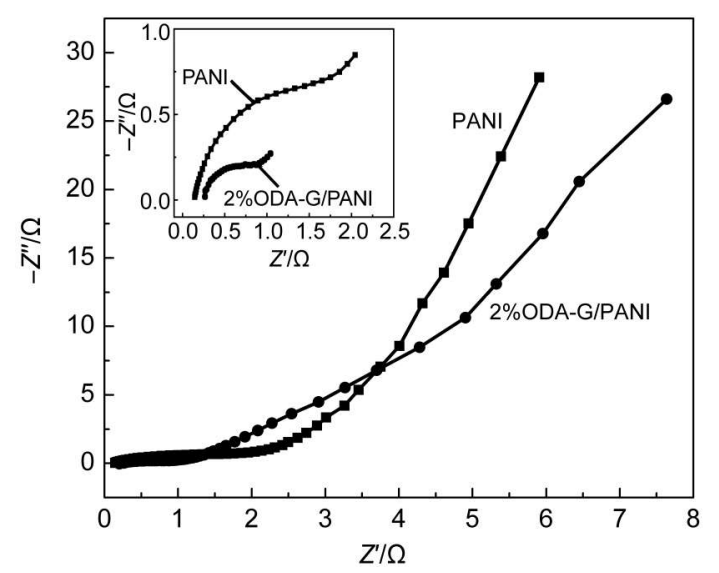

图 10 PANI 和 $2 \%(w)$ ODA-G/PANI 复合材料在 $10^{-2}-10^{5}$ $\mathrm{Hz}$ 频率范围内的电化学阻抗图

Fig.10 Electrochemical impedance spectroscopies of PANI and $2 \%(w)$ ODA-G/PANI composite in the frequency range of $10^{-2}-10^{5} \mathrm{~Hz}$ 


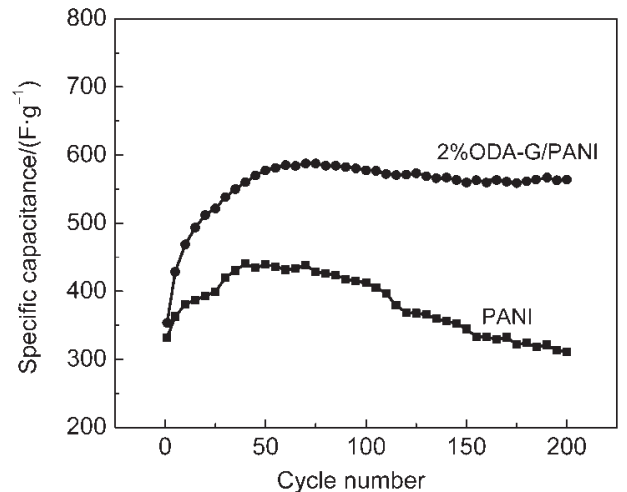

图 11 PANI 和 $2 \%(w)$ ODA-G/PANI 复合材料在电流密度为 $10 \mathrm{~A} \cdot \mathrm{g}^{-1}$ 时的循环性能

Fig.11 Cycling performance of PANI and 2\%(w)ODA-G/ PANI composite at a current density of $10 \mathrm{~A} \cdot \mathrm{g}^{-1}$

时, 比电容值仍保持在 $620 \mathrm{~F} \cdot \mathrm{g}^{-1}$, 说明其具有良好 的倍率特性.

图 10 为 PANI 与 $2 \%(w) \mathrm{ODA}-\mathrm{G} / \mathrm{PANI}$ 复合材料 在测试频率范围 $10^{-2}-10^{5} \mathrm{~Hz}$ 内的交流阻抗图. 两条 交流阻抗曲线在低频区的斜线斜率反映出二者具 有良好的电容性. 如插图所示, 高频区 $2 \%(w)$ ODAG/PANI 的圆弧半径小于 PANI 的圆弧半径, 说明 $2 \%$ ODA-G/PANI 的电荷转移阻值要小, 即 ODA-G 的加 入对电化学过程中电荷转移阻值的减小有所贡献 ${ }^{11}$.

图 11 为纯 PANI 和 $2 \%(w)$ ODA-G/PANI 复合材 料在电流密度 $10 \mathrm{~A} \cdot \mathrm{g}^{-1}$ 下的循环性能曲线. 从图中 可以看出, 经过相同的循环次数之后, $2 \%(w) \mathrm{ODA}-$ G/PANI 的比电容值远大于 PANI 的, 说明 ODA-G 的 加入大大改善了电极材料的比电容. 起始循环阶 段, 比电容值较低是由于电极材料未完全参与反 应. 随着循环次数的增加, PANI 电极材料的比电容 衰减较快, 经 200 次循环后比电容仅为最高比电容 的 70\%, 而 ODA-G/PANI 电极材料的比电容衰减较 慢, 最高比电容保持率为 $96 \%$, 说明 ODA-G/PANI 具 有良好的循环特性, ODA-G 的添加还有效地提高了 电极的使用寿命。

\section{4 结 论}

本文通过用简便的溶液混合法制备了 ODA-G/ PANI 纳米复合电极材料, 发现 ODA-G 较好地嵌入 到纳米 PANI 之间或包裹在纳米 PANI 表面, 可以有 效地发挥二者的协同作用且有益于复合材料结构 的稳定. 电化学性能测试表明, 在电流密度为 $1 \mathrm{~A} \cdot \mathrm{g}^{-1}$ 时, $2 \%(w)$ ODA-G/PANI复合材料的比电容为 $787 \mathrm{~F}$. $\mathrm{g}^{-1}$, 远远高于纯 PANI 的值 $\left(426 \mathrm{~F} \cdot \mathrm{g}^{-1}\right)$, 经过 200 次循
环后比电容仍能保持最高比电容的 $96 \%$. 另外, ODA-G/PANI 复合材料具有好的功率特性, 是理想 的超级电容器用电极材料. 本文报道的简便方法有 可能广泛应用于未来石墨烯基电极复合材料的大 规模制备.

致谢: 本文的 TEM 分析得到了钢铁研究总院袁泽明博士 的协助, 本科生俞慧涛同学参与了部分实验工作, 在此一并 致谢!

\section{References}

(1) Yang, G. W.; Xu, C. L.; Li, H. L. Chem. Commun. 2008, 6537.

(2) Brownson, D. A. C.; Kampouris, D. K.; Banks, C. E. J. Power Sources 2011, 196, 4873. doi: 10.1016/j.jpowsour.2011.02.022

(3) Dikin, D. A.; Stankovich, S.; Zimney, E. J.; Piner, R. D.; Dommett, G. H. B.; Evmenenko, G.; Nguyen, S. T.; Ruoff, R. S. Nature 2007, 448, 457. doi: 10.1038/nature06016

(4) Stoller, M. D.; Park, S. J.; Zhu, Y. W.; An, J.; Ruoff, R. S. Nano Lett. 2008, 8, 3498. doi: 10.1021/n1802558y

(5) Wu, H. Y.; Wang, H. W. Acta Phys. -Chim. Sin. 2013, 29, 1501. [吴红英, 王欢文. 物理化学学报, 2013, 29, 1501.] doi: 10.3866/PKU.WHXB201304241

(6) Xu, G. Y.; Ding, B.; Nie, P.; Luo, H. J.; Zhang, X. G. Acta Phys. -Chim. Sin. 2013, 29, 546. [徐桂银, 丁 兵, 聂 平, 骆 宏钧, 张校刚. 物理化学学报, 2013, 29, 546.] doi: 10.3866/ PKU.WHXB201301081

(7) Wang, H. Z.; Gao, C. X.; Zhang, P.; Yao, S. W.; Zhang, W. G. Acta Phys. -Chim. Sin. 2013, 29, 117. [王宏智, 高翠侠, 张 鹏, 姚素薇, 张卫国. 物理化学学报, 2013, 29, 117.] doi: 10.3866/PKU.WHXB201210234

(8) Sun, Y.; Wu, Q.; Shi, G. Energy Environ. Sci. 2011, 4, 1113. doi: 10.1039/c0ee00683a

(9) Gómez, H.; Ram, M. K.; Alvi, F.; Villalba, P.; Stefanakos, E.; Kumar, A. J. Power Sources 2011, 196, 4102. doi: 10.1016/j. jpowsour.2010.11.002

(10) Yan, J.; Wei, T.; Shao, B.; Fan, Z.; Qian, W.; Zhang, M.; Wei, F. Carbon 2010, 48, 487. doi: 10.1016/j.carbon.2009.09.066

(11) Zhang, K.; Zhang, L. L.; Zhao, X. S.; Wu, J. Chem. Mater. 2010, 22, 1392. doi: $10.1021 / \mathrm{cm} 902876 \mathrm{u}$

(12) Liu, Y.; Deng, R.; Wang, Z.; Liu, H. J. Mater. Chem. 2012, 22, 13619. doi: $10.1039 / \mathrm{c} 2 \mathrm{jm} 32479 \mathrm{~b}$

(13) Lai, L.; Yang, H.; Wang, L.; Teh, B. K.; Zhong, J.; Chou, H.; Chen, L.; Chen, W.; Shen, Z.; Ruoff, R. S.; Lin, J. ACS Nano 2012, 6, 5941. doi: 10.1021/nn3008096

(14) Wang, D. W.; Li, F.; Zhao, J.; Ren, W.; Chen, Z. G.; Tan, J.; Wu, Z. S.; Gentle, I.; Lu, G. Q.; Cheng, H. M. ACS Nano 2009, 3, 1745. doi: 10.1021/nn900297m

(15) Zhang, Q.; Li, Y.; Feng, Y.; Feng, W. Electrochim. Acta 2013, 90, 95. doi: 10.1016/j.electacta.2012.11.035

(16) (a) Luo, B.; Liu, S.; Zhi, L. Small 2012, 8, 630. 
(b) Singh, V.; Joung, D.; Zhai, L.; Das, S.; Khondaker, S. I.; Seal, S. Prog. Mater. Sci. 2011, 56, 1178. doi: 10.1002/smll.v8.5

(17) Li, W.; Tang, X. Z.; Zhang, H. B.; Jiang, Z. G.; Yu, Z. Z.; Du, X. S.; Mai, Y. W. Carbon 2011, 49, 4724. doi: 10.1016/j. carbon.2011.06.077

(18) Kovtyukhova, N. I.; Ollivier, P. J.; Martin, B. R.; Mallouk, T. E.; Chizhik, S. A.; Buzaneva, E. V.; Gorchinskiy, A. D. Chem. Mater: 1999, 11, 771. doi: 10.1021/cm981085u

(19) Jeong, H. K.; Lee, Y. P.; Jin, M. H.; Kim, E. S.; Bae, J. J.; Lee, Y. H. Chem. Phys. Lett. 2009, 470, 255. doi: 10.1016/j. cplett.2009.01.050

(20) Stankovich, S.; Dikin, D. A.; Piner, R. D.; Kohlhaas, K. A.; Kleinhammes, A.; Jia, Y.; Wu, Y.; Nguyen, S. T.; Ruoff, R. S. Carbon 2007, 45, 1558. doi: 10.1016/j.carbon.2007.02.034
(21) Li, W. P.; Liu, S. H.; Duan, Y. P.; Li, C. M. J. Mater. Sci. Technol. Eng. 2007, 25, 376. [李伟平, 刘顺华, 段玉平, 李长 茂. 材料科学与工程学报, 2007, 25, 376.]

(22) Malarda, L. M.; Pimenta, M. A.; Dresselhaus, G.; Dresselhaus, M. S. Phys. Rep. 2009, 473, 51. doi: 10.1016/j. physrep.2009.02.003

(23) Ferrari, A. C. Solid State Commun. 2007, 143, 47. doi: 10.1016/j. ssc. 2007.03 .052

(24) Ganesan, R.; Shanmugam, S.; Gedanken, A. Synth. Met. 2008, 158, 848. doi: 10.1016/j.synthmet.2008.06.001

(25) Cochet, M.; Louarn, G.; Quillard, S.; Buisson, J. P.; Lefrant, S. J. Raman Spectrosc. 2000, 31, 1041.

(26) Stoller, M. D.; Ruoff, R. S. Energy Environ. Sci. 2010, 3, 1294. doi: $10.1039 / \mathrm{c} 0 \mathrm{ee} 00074 \mathrm{~d}$ 